Original Reasrch Paper

\title{
Design and Analysis of a Lunar Lander's Landing System
}

\author{
${ }^{1}$ Anurag Talekar, ${ }^{2}$ Ajinkya Dabholkar, ${ }^{3}$ Ajinkya Thombe, ${ }^{4}$ Karan Jethwa and ${ }^{5}$ Nandkumar Bhopale \\ 1,2,3,4 Department of Mechanical Engineering, MGM College of Engineering and Technology, Navi Mumbai, India \\ ${ }^{5}$ Department of Mechanical Engineering, MGM College of Engineering and Technology, Navi Mumbai, India
}

\author{
Article history \\ Received: 05-05-2021 \\ Revised: 23-05-2021 \\ Accepted: 27-05-2021 \\ Corresponding author: \\ Nandkumar Narayanrao \\ Bhopale \\ Department of Mechanical \\ Engineering, MGM College \\ of Engineering and \\ Technology, Navi Mumbai, \\ India \\ Email: bhopalen@yahoo.com
}

\section{Introduction}

Along with the progress of sciences and technologies, a lot of exploration is taken in many countries or organizations in succession. Lunar, the natural satellite of the earth, is a focus of space discovery because of its abundant resources and high value in use. The Chandrayaan-2 spacecraft to the moon was a composite module mission consisting of Orbiter, Lander and Rover. The combined stack was then inserted into a lunar orbiter for a soft landing on a site near the south polar lunar surface. The overall objective of Chandrayaan-2 was to collect samples from the lunar surface and analyze them on-site, relaying data to Earth via the orbiter. Unfortunately, Vikram lander lost control $500 \mathrm{~m}$ short of the lunar surface and crashed (Jones, 2015; Sahinoz, 2012; Battrick et al., 1992; Huang et al., 2015; Borowski et al., 2018). The glitch was unexpected since the software was functioning well throughout the trial period. The Vikram lander successfully glided from a height of 30 to $5 \mathrm{~km}$ after this rough braking, the lander experienced trouble during the final braking, the final stage in which the lander operated only one of its thrusters and slowed down to just $146 \mathrm{~m} / \mathrm{s}$. the lander veered off its trajectory and crashed $750 \mathrm{~m}$ away from the indented landing spot. The impact of the crash damaged the machinery onboard and the lander went incommunicado (Carpenter et al., 2010; Jinbao and Hong, 2008; Wang et al., 2019; Collier et al., 2011; Glenar et al., 2011; Huang et al., 2016).

To ensure arresting the spacecraft safely, the analysis of touchdown dynamics during the landing stage was important. The Chandrayaan- 2 consisted of a lunar orbiter and also included the Vikram lander and therefore the Pragyan lunar rover. The main scientific objective was to map and study the variations in lunar surface composition, also because of the location and abundance of lunar water. The descent board computers on lander, with mission are unable to form corrections. The initial descent was considered within mission parameters, passing critical braking procedures needless to say, but the lander's trajectory began to deviate at about $2.1 \mathrm{~km}(6,900 \mathrm{ft})$ above the surface (Yu and Luo, 2019; Kosambe, 2019; Nie et al., 2019). A mission to land on the moon failed when the lander, of the Chandrayaan-2 mission, crashed on the lunar surface.

Due to the absence of atmosphere on the moon, it is difficult for a lander to have a soft touchdown using nonconventional technology. Landing on the lunar surface is divided into three parts i.e., the de-boost phase, rough braking, fine braking. The first two sequences are normal and the probability of the failure of the mission is $10-20 \%$ as it all depends on the boosters onboard. During the final phase of fine braking, the reverse boosters are 
fired which decelerates the lander's speed from 86 to 60 $\mathrm{m} / \mathrm{s}$ after which the lander experiences a free fall. The fine braking starts from the approx altitude of $400 \mathrm{~m}$ and the force gained by the lander exerted on the landing system (Ding et al., 2019).

If the landing gears of a lander could not bear the above-mentioned force, then it might face the breaking of the landing gears. This may damage the lander or it may topple also. Lander of the mission Chandrayaan-2 had a similar failure. The onboard radar failed to provide the accurate altitude and according to it, the booster calibrated itself to the wrong data. Due to this, a lander experienced a free fall with a change in orientation. This crash would have been avoided if the lander's landing system would have been designed beyond experienced value.

As the space industry is developing very rapidly from the past few years. The curiosity of unriddling the enigma of the existence of our home planet has led mankind to resolve the unsolved mysteries of the universe. Recently, the rover has made its place intrinsic in such observation expeditions as it can examine the moon surface very attentively. But, employing the rover on such lunar surface is a very intricate task as the lander carrying the rover has to land on the lunar surface very softly.

In the open literature, it is found that, for the deployment of a rover with the aid of a lander design, proper working of a landing gear and its control system are one of the very crucial tasks. There are few technologically advanced control systems that can be used to navigate the lander through space. Also, scientifically feasible landing gear constructions are considered to achieve the soft landing of the lander.

Few authors have attempted to propose a possible solution for adaptive landing gears by introducing hydraulic systems, intelligent materials and pyrotechnic devices. Some of them also proposed a theory of balloon or parachute landers. But such type of landers is ineffective in the thin atmosphere of the moon. The past researchers have tried to improve the stability of the module by optimizing pivot points in construction and keeping the very common 4-legged pod structure constant. It is found that consistency of the structure limits the implementation of the new ideas to improve stability.

It is understood from the literature review that, very small work has been done on improving the stability and improving landing of lunar lander. Moreover, there is not adequate literature that explores its applications on land to improve stability and check the possibilities of airbags/cushioning.

The proposed work mainly aims to significantly reduce the failure of the lunar lander mission which occurs mainly due to failure in the landing gear system. In this study, an attempt is made to significantly reduce the crushing force acting on the lander during freefall by applying the scientific data available and smart materials for the same. The novel method is used which is different from the structures that have been found in past research. The motors are employed to give stable horizontal position to the lander. This would help the module to achieve the required horizontal slope for the successful deployment of the rover. Also, the lander is capable of reading the lunar bumps and arranging its legs according to the need. It can also be tested for its landing on the declining lunar surface while keeping the horizontal level of the lander constant. Such abovementioned features of this module grant a huge benefit over conventional lander structures.

\section{Design and Analysis}

\section{A. Design Parameters}

The design of the lander is decided according to the requirement of the mission assigned which includes lander properties and lunar conditions. However, another factor that affects the weight of the lander is the space transportation system available with the ISRO. Currently, GSLV MK3 is the most powerful rocket in ISRO inventory. The GSLV MK3 with payload is shown in Fig. 1. This rocket is capable of placing a mass of $3,400 \mathrm{~kg}$ into the earth's orbit thus, making it sufficient to successfully place the lander into the lunar orbit followed by the landing phase. Such a mission generally includes an orbiter, lander and rover. However, at the time of landing the lander will weigh less than $1400 \mathrm{~kg}$ as the fuel stored will be used for its commute through vacuum i.e., space and during the process of landing a desirable location. The dynamic envelope of the launch vehicle (GSLV MK3) is approx. $4 \mathrm{~m}$ in diameter, which means the legs of the lander must be within $4 \mathrm{~m}$ to accommodate the vehicle pod. The hazard avoidance system capable of detecting a rock larger than $25 \mathrm{~cm}$ as smaller than this would not affect lander position which might lead to toppling. The system gets activated when it detects the slope ranging from $9^{\circ}$ to $10^{\circ}$ angles at the time of landing. The lunar gravity is $1.63 \mathrm{~m} / \mathrm{s}$ i.e., $1 / 6$ th of the earth's gravity.

For a safe landing, a lander should normally experience a vertical velocity of $2 \mathrm{~m} / \mathrm{s}$ with zero horizontal velocity. Also, to conserve this lander and to minimize the toppling effect on the lander, the worst scenario of $4 \mathrm{~m} / \mathrm{s}$ vertical and $1 \mathrm{~m} / \mathrm{s}$ horizontal is selected.

The temperature has a wide range depending on the location of the crater to the surface under a shadow it usually ranges from $100^{\circ}$ to $120^{\circ} \mathrm{C}$. The effect of such temperature on this design is neglected (eliminated during material selection.)

Soil bearing strength increases as we go deeper into lunar soil starting from $0.2 \mathrm{~N} / \mathrm{cm}^{2}$ at $1-2 \mathrm{~mm}$ and reacting $5.5 \mathrm{~N} / \mathrm{cm}^{2}$. 


\section{B. Main Body}

A legged and a pod lander are generally used as the main structure of the lander. There are extremely rare chances for the lander's soft landing when it has been decelerated from the speed of 80 to 5-7 kmph. In such a case, we cannot deny the possibility of the toppling of the lander. To achieve a soft landing, the lander should be light in weight and at the same time it should possess the strength to survive the freefall of the $30 \mathrm{~m}$ on the lunar surface. The design concept of the landing gear system is made straight from the simplest configuration to a more complex structure.

This section examines the development of the lunar lander alongside its parts. The above Fig. 2 illustrates the idea of newly designed lander module.

The lander is an enhanced six-legged plan where every leg is comprised of a honeycomb damper. The principle design of this lander is hexagonal with every leg mounted on each side of the hexagonal undercarriage. The legs are given foot expansions to acquire a solid grasp on the lunar surface and $360^{\circ}$ encompass assurance. The parts are amassed along with the assistance of rivets. Rivet 1 and Rivet 2 are utilized with a motor to give the free developments of the legs. This whole construction is produced using the titanium composite Ti-8AI-1Mo-1V. The empty space on the hexagonal surface will be utilized to mount the payload to be carried as shown in Fig. 3 .

\section{Landing Gear}

In the design of lunar lander, the landing gear system is considered to be one of the most important components. To position the above-mentioned lunar module firmly on the lunar surface, we have designed a novel leg. The lunar struts design should be such that it must absorb the impact energy as much as possible to protect the payload (rover) and onboard instruments when the lunar module touches the lunar soil. While these legs while functioning cumulatively will provide the module-required landing position and safety from the toppling. Thus, establishing desirable physical conditions for the rover to roll out securely. These legs are liked to be inflexible because of energy retention proficiency and strength against unsure landing conditions. The fundamental body is a mounting stage for different distinguishing instruments and control subsystems. The entirety of the arrival gears, with a similar setup and size are made out of one primary strut, one secondary strut and one footpad. The association between struts is acknowledged through an all-inclusive joint, so as among struts and fundamental body, while the footpad is associated with the primary strut by the rotating appendage. This construction is well illustrated in Fig. 4 below.

These legs are utilized for force absorption to limit the G-force experienced by the spacecraft where the leg supports the lander structure and provides clearance to avoid a collision.
Also, the legs have to be introduced with the folding mechanism and they are stood during launch and flight and deployed after detachment from the rover. These legs will have a vertical motion to get adjusted according to the demand as the lunar surface is very rutted. The extended supports will drop down onto the lunar surface immediately after the six legs hit the ground. This piercing action of the legs will assure a firm foundation of the lander. Eventually, the lander and the payload will be secured and ready to carry out the next operation i.e., deployment of the rover.

As shown in the Fig. 5 above this leg is also employed with a special damper. The damper performs a crucial part in the underlying dynamic and acoustic exhibition of the lander. A large portion of the crushable power following up on the lander will be consumed by this damper. It almost ingests 70-80\% of the power following up on it and the leftover power following up on a similar will be consumed by the construction of the lander.

\section{Damper Design}

Energy can neither be created nor be destroyed, it can only be converted from one form to each another. It is the most basic law of nature. Hence in vehicle dynamics, it is necessary to dissipate the energy absorbed or store this energy by employing absorbent materials when the vehicle hits a rock bottom. And thus, the vehicle experiences a cushioning effect (Montgomery, 1997).

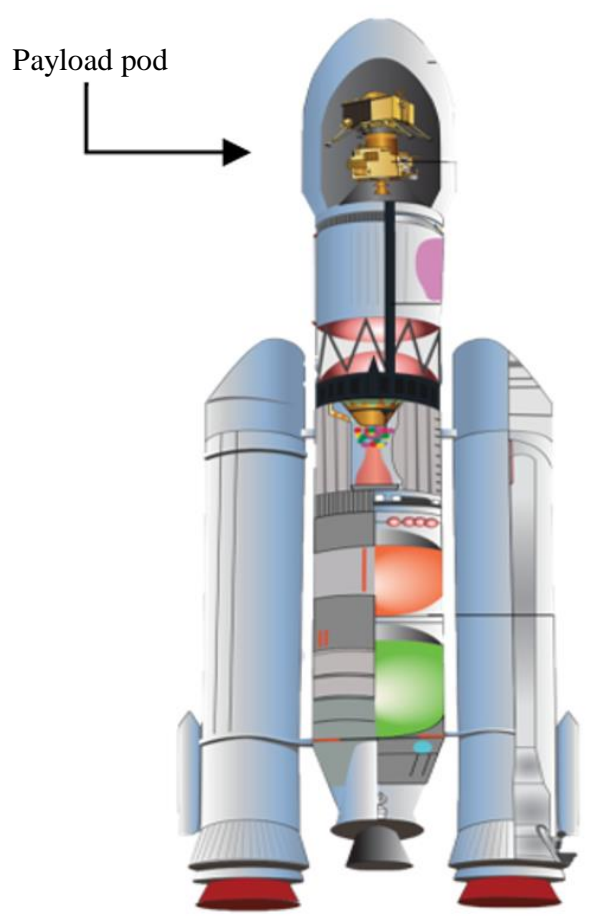

Fig. 1: GSLV MK3 with payload (From Chandrayaan-2: India's Second Lunar Exploration Mission. -2019) 


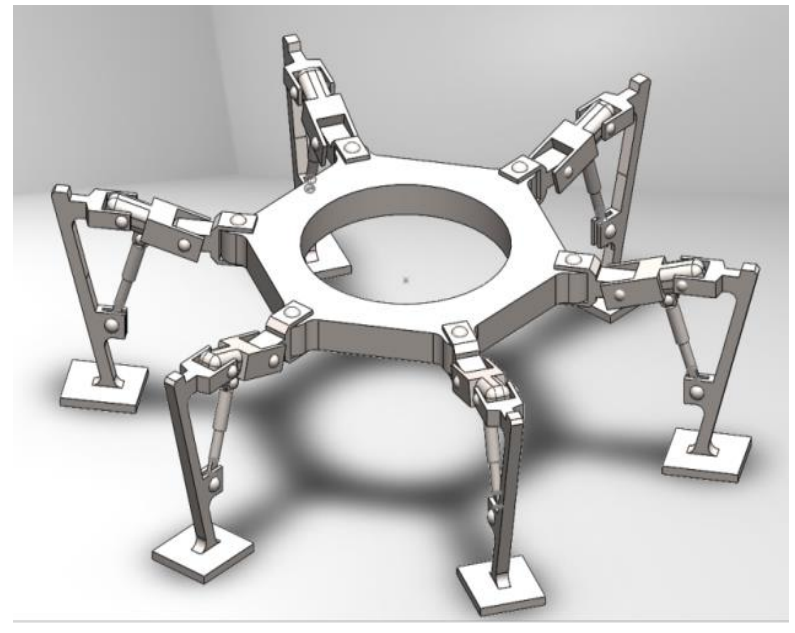

Fig. 2: Computerized 3D model

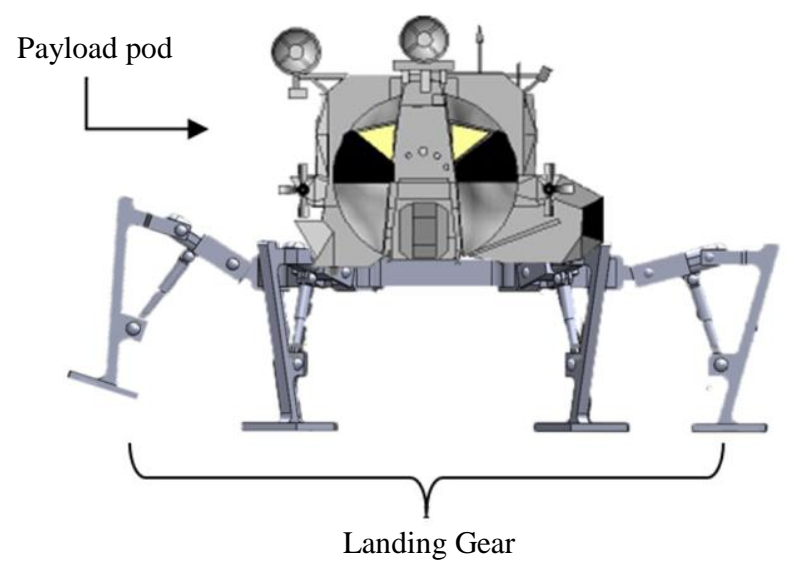

Fig. 3: Conceptualization

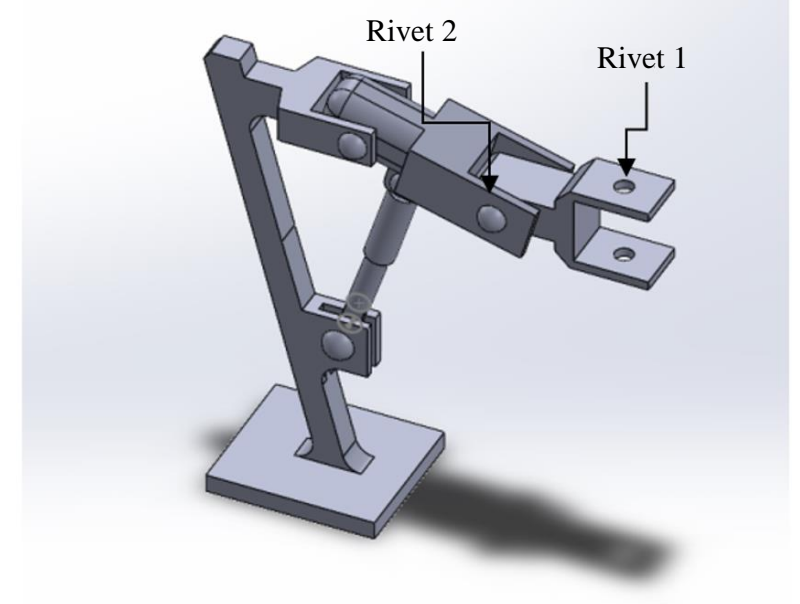

Fig. 4: Construction of novel leg

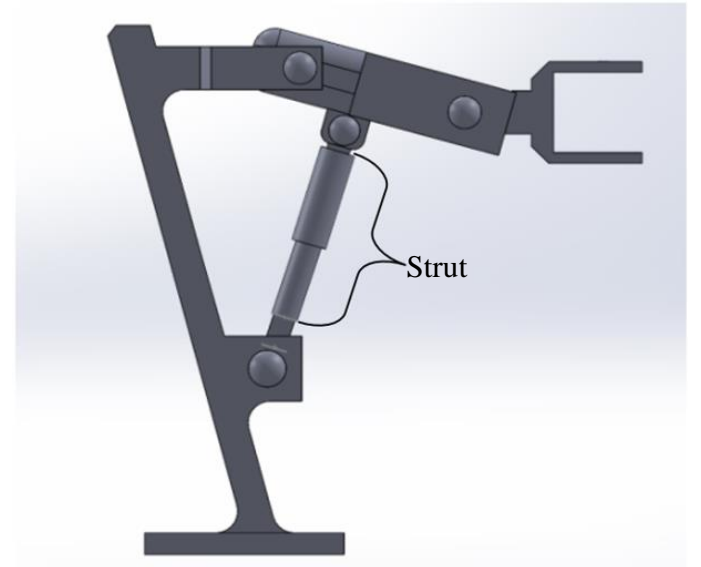

Fig. 5: Strut Placement

It is well-known fact that dampers are used to dissipate the kinetic energy produced by a vehicle and provide a cushioning effect to the same. The ordinary damper absorbs this energy and releases it by retaining its original form. This retention of the form or elastic nature of the damper may cause some serious issues when we are considering a lunar module landing on the lunar surface having less gravity. The elastic nature is more likely to make the lander unstable even after the perfect touchdown and eventually, it might get toppled. For this reasons, we decide to utilize an energy absorbent system which will consequent in a stable landing by locking the landing gears in real-time after hitting the lunar surface.

Apollo-II, the first spacecraft that brought men to the lunar surface, had used a honeycomb shock absorber. According to the study, the honeycomb structure is the most efficient and reliable geometry to provide energy absorption and load protection to the structure leading to intentionally plastic deformation of a designated element. When loaded, the respective element deforms plastically and proportionally to stroke. The capacity of energy absorption is determined by the integration of the absorbed force $F(s)$ over the stroke, thus:

$E=\int f(s) d s$

Hence, to achieve our goal, we decided to employ the honeycomb cartilage dampers as shown in Fig.6.

Aluminum is a directional lightweight energy absorbent material which provides a constant force during the stroke. It can compress upto $70-80 \%$ of its original length. The only disadvantage of using honeycomb is that there is a drastic loss of strength with increasing crush angle, shown in Fig. 7.

This is overcome by the piston in an internal cylinder. This strut comprises one inner cylinder (piston), one external cylinder, three base plates placed in between honeycomb cartilage and two honeycomb cartilages (variable hexagonal dia.) all shown in the Fig. 8 and 9. The strut is attached to the lander leg with the help of 
rivets. The following list suggests the materials used for the above-mentioned components-:

a. Inner and external cylinder - 201 Annealed Stainless Steel (SS)

b. Base plates-Aluminum Bronze

c. Honeycomb Cartilage-Aluminum alloy series 3000/3003/3005/3103/3104

The struts we designed to have two honeycombs of different sizes to attain different crush strength and are separated by base plate as shown in Fig. 9. The first stage of the honeycomb has small crush strength and that second stage has larger crush strength. The main motto of using variable cells is that it can reduce the peak acceleration as well as absorb the whole impact energy.
When the lander hits the ground, the energy gets transferred from the foot extension to the legs and eventually the cell with small crush strength absorb the whole impact energy and the damper which then starts actuating. This energy from the ground is now getting absorbed by the cartilage. When the load increases, the cartilage starts to crumple to absorb the maximum load. At a particular point, the load produced so far becomes insufficient to further compress the structure and crumpling of the cartilage stops. This crumpling is plastic in nature and hence it doesn't urge to regain its original form. Due to this irreversible phenomenon, the damper gets locked and a stable landing is achieved by the successful employment of the honeycomb cartilage dampers. The above Fig. 10 represents the initial, intermediate and final state of the cartilage for better understanding.

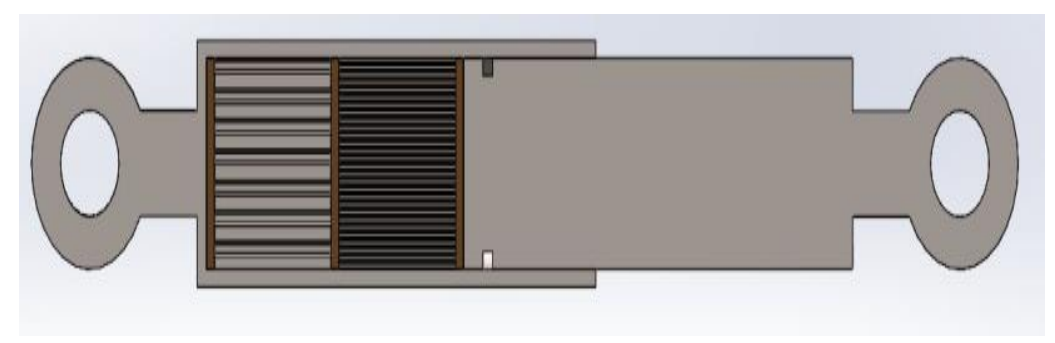

Fig. 6: Structure of honeycomb cartilage damper
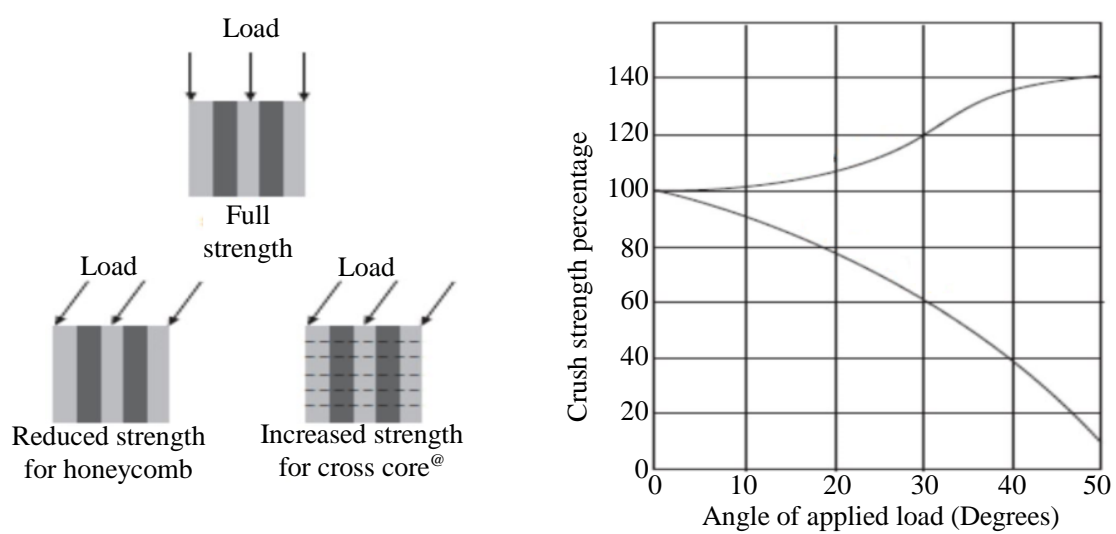

Fig. 7: Honeycomb strength vs. crush angle (Landing Gear Design and Stability Evaluation of a Lunar Lander for Soft Landing. PA 15213)

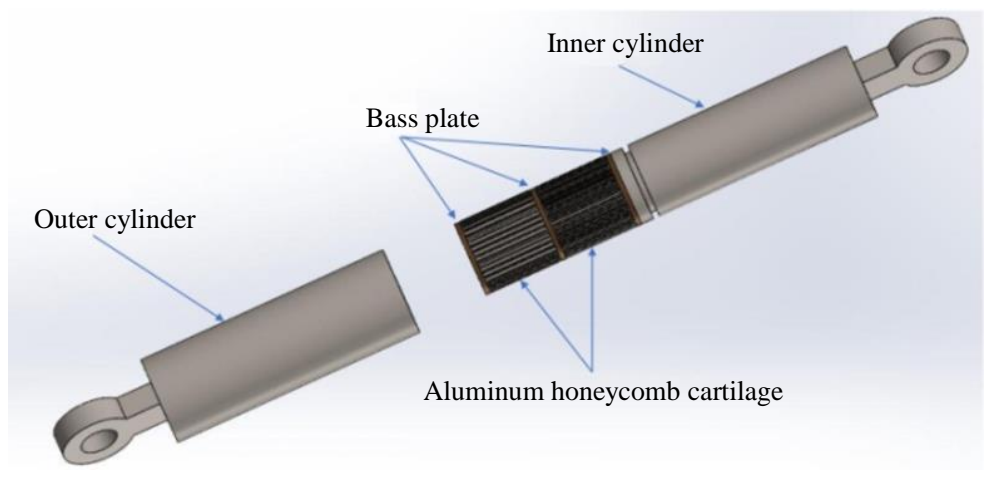

Fig. 8: Exploded view of honeycomb cartilage damper 


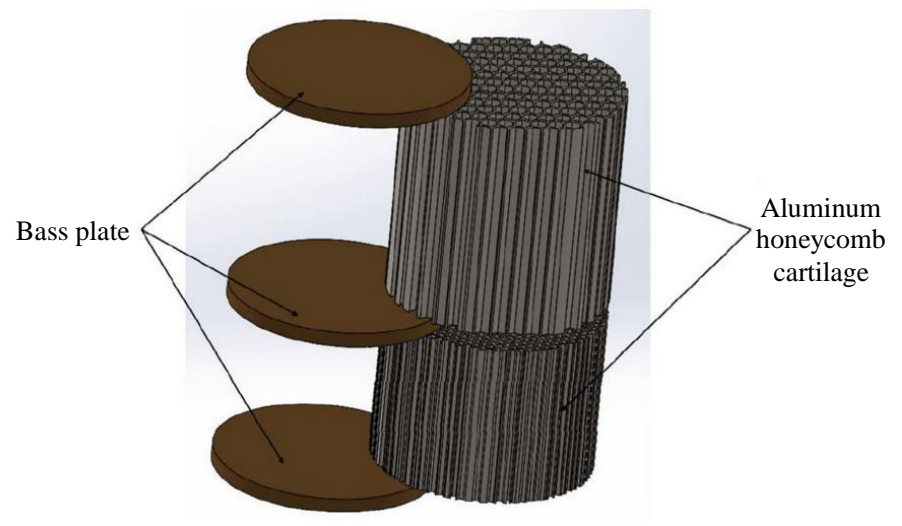

Fig. 9: Aluminum honeycomb cartilage setup
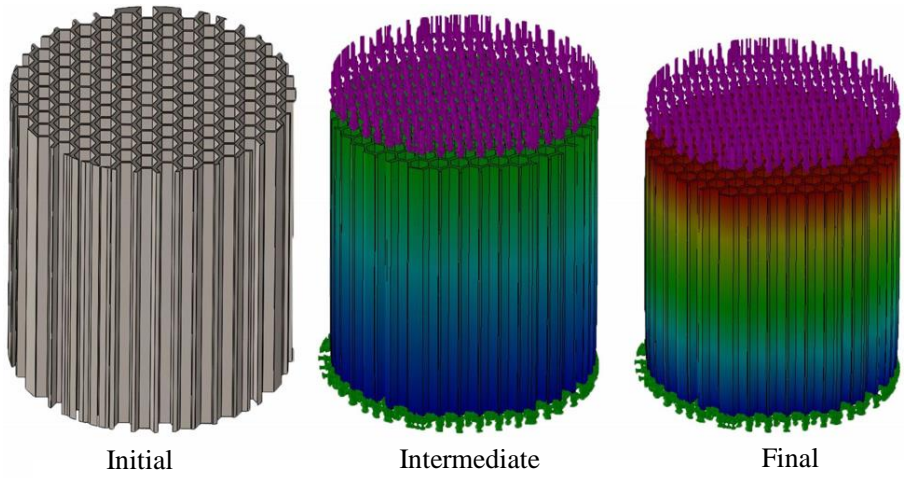

Fig. 10: Honeycomb cell under variable load

\section{Motor Selection}

For the selection of electric motor and the accessibility on the lookout, there are 4 standard sorts of electric motors that are reasonable for space applications viz:
a. AC Induction Motors
b. Brush Direct Current (BDC) Motors
c. Brushless Direct Current (BLDC) Motors
d. Stepper Motors

Among the previously mentioned types, BLDC motor are essentially preferred in different space programs. The choice of such motors is justified below. BLDC motor makes them out qualifying benefits over other motors. As we think about the heaviness of the entire lander module and the lunar surface conditions, we should utilize motors with higher torque which can work effectively taking care of the enormous weight. The output torque of the BLDC motor is a lot higher than the BDC motor. BLDC motor delivers roughly twofold the output torque than the equivalent size of the BDC motor.

At the point when the motor rotates, it produces high torque which ought to be scattered for its smooth working. BLDC motor has its winding on the stator rather than the rotor which works with heat dispersal. As the name recommends, BLDC motor doesn't employ brushes. Due to this the life of the motor increases. It has exceptionally high productivity. Considering, the lunar surface has no environment. In other words, the working medium for the motor will be a vacuum. BLDC motor is tremendously viable in vacuum conditions.

After thinking about all the above things, we have selected to go for the BLDC motor. By employing this motor, the rotation of the legs pivoted at rivet 2 is achieved. The setup is thus moved vertically downward and upward according to the demand of the lunar surface. For the ease in operation, the movement of the same is made constraint until those legs do not tangle with each other. The arrangements of the same are shown in the above Fig. 11.

\section{Working}

In the above section, the components and the function of the same are explained. The working of the whole module is depicted in Fig. 11 is assembled with the rivets and other suitable fasteners used in space programs.

The initial landing procedure starts $100 \mathrm{~km}$ away from the landing site where the thruster starts firing in the 
opposite direction of the lander. Due to this De-boost phase the lander goes under de-orbiting maneuvers. When the lander gets separated from the orbiter, the mounted control system takes over and pilots the further route for the lander. The onboard sensors are works as the brain and neural system of the lander. The data provided by the sensors is used to maintain the required speed and the position of the lander with respect to horizontal as well as vertical axis. The radar placed directly beneath the footpad of the lander gets activated and anticipates accordingly. Based on the measured vertical distance from the ground directly beneath the feet to skid pad as illustrated in Fig. 12, a controller is used to analyze the data and control the horizontal velocity to avoid any geek or rock.

This data received from the radar is analyzed and used to control the adjustment of the acting cylinder. The main aim of the adjustment is to ensure that six feet touch the oblique terrain at the same time so that the capsule remains horizontally parallel to the ground and intact during landing. The radar reads this information and then makes predictions as needed. It will then determine the final landing point and leg position to stay in horizontal level without toppling. When the lander hits the ground, the force will propagate from the ground to the skid pads, then to the lower leg part and finally to the damper. Then, the damper will absorb the impact caused by the landing. The honeycomb structure consumes the applied force by getting crushed vertically. At a particular point, this force won't be sufficient to further crush the structure and the crumpling will stop. Thus the lander achieves its stability. The slop landing controller decision is shown in Fig. 13.

\section{G. Calculations and Analysis}

A lunar lander experiences a free fall from a height of a 30meters. This sudden movement besides the subsequent hitting of the lander to the ground would cause damage to the lunar module payload. Given the available information, the maximum weight of the lander is 1.5 tons with a vertical velocity of $5 \mathrm{~m} / \mathrm{s}$ (maximum). The stress analysis of honeycomb cartilage damper is illustrated in Fig. 14. Here no horizontal velocity is acting on the lander so the vertical velocity would be lower than $5 \mathrm{~m} / \mathrm{s}$. Following the hitting of the vehicle to the ground with a velocity of $5 \mathrm{~m} / \mathrm{s}$, the average impact force of $3770 \mathrm{~N}$ will be applied to lunar lander. The displacement analysis of honeycomb cartilage damper is depicted in Fig. 15.

Mathematically, it can be represented as follows we are considering that the weight of the lander on the earth will not exceed more than $1500 \mathrm{Kg}$, but to acquire the actual mass of the lander, we need to divide it by the gravitational force applied from the earth i.e., 9.81 $\mathrm{m} / \mathrm{s}^{2}$. Hence:

$m=\frac{w}{g}$

$\therefore m=\frac{1500}{9.81}=152.90 \mathrm{~kg} \approx 153 \mathrm{~kg}$

- To simplify the calculations $155 \mathrm{~kg}$ is considered

- According to the work-energy principle

- $\quad$ Average impact force $=m g h$

- Where,

- Mass of the lander $=m=155 \mathrm{~kg}$

- Acceleration due to gravity on moon $=g=1.62 \mathrm{~m} / \mathrm{s}^{2}$

- $\quad$ Free fall height $=h=10 \mathrm{~m}$

- $\therefore$ Average impact force $=155 \times 1.62 \times 10=2511 N$

- Considering the Factor of Safety $=1.5$

- Average impact force $=2511 \times 1.5=3766.5 N \approx$ $3770 N$

- Therefore, the force of 3770 Newton has been considered while landing.

- These values may be used for the analysis and simulation purpose of the honeycomb damper.

- Fig.14 and Fig. 15 assembles the cad models of landing gear (honeycomb cartilages Al 1.7-3105$.0393 \mathrm{~N})$.

- $\quad$ These components are analyzed on SolidWorks 2020. Model shows quadratic displacement behavior.

- Number of elements in model: 261298

- Number of nodes in model: 473459

- Material Properties:

- Material: Al

- Poisson's Ratio: 0.22

A static load of $1000 \mathrm{~N}$ is applied to honeycomb cartilages. A maximum stress $(\sigma)$ of $1.832 \mathrm{e}+06 \mathrm{~N} / \mathrm{m}^{2}$ resulting in the displacement of $3.946 \mathrm{e}-0433 \mathrm{~mm}$ was observed.

All the component shown in Fig. 8 are assembled to form damper (landing gear) and its analysis was done on SolidWorks as shown in Fig. 16 (after deformation):

- Jacobian points of high quality mesh: 16 points

- Number of elements in model: 166,889

- Number of nodes in model: 301211

- Material properties:-

- Material: SS (ferritc), $\mathrm{Al}, \mathrm{AlBr}$

- Poisson's Ratio: 0.3, 0.22, 0.28

Stresses and Displacement data was observed in FEA shown in Table 1. 


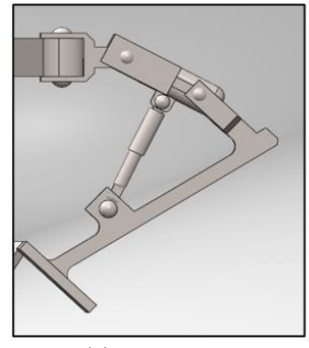

(a)

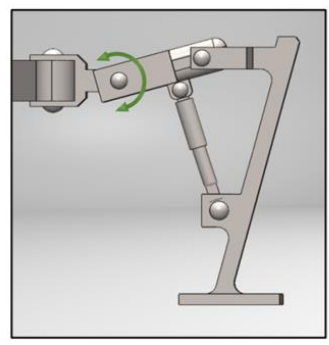

(b)

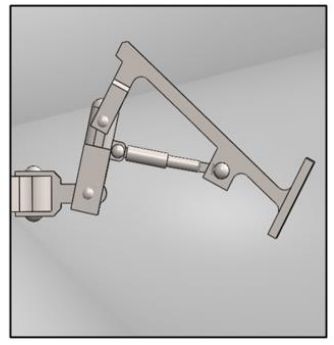

(c)

Fig. 11: Landing Gear actuation using BLDC motor; (a) Downward Rotation; (b) Idle Position; (c) Upward Rotation At an all titude of $100 \mathrm{~km}$ lander changes trajectory
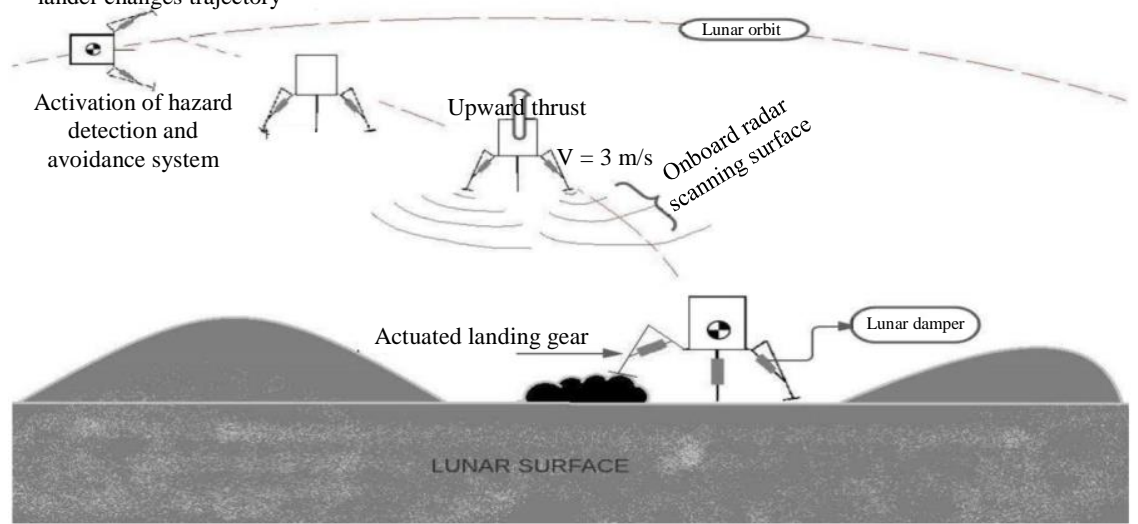

Fig. 12: Conceptual framework

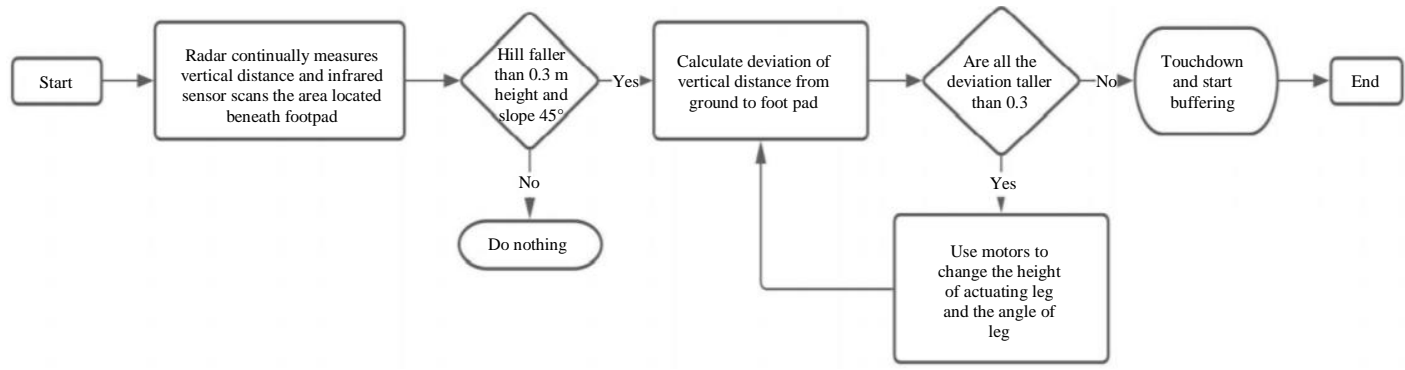

Fig. 13: Slope landing controller decision flow chart

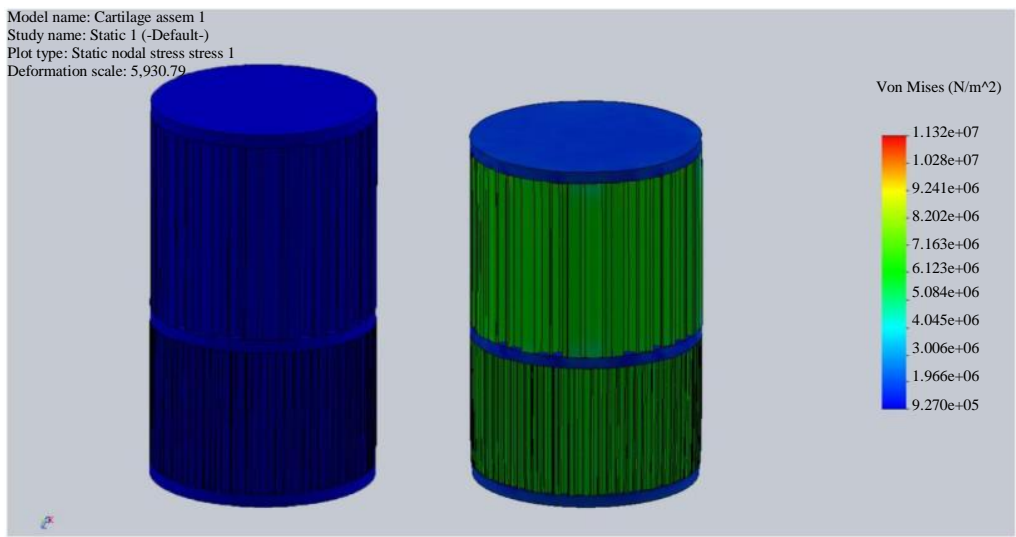

Fig. 14: Stress analysis of honeycomb cartilage damper 


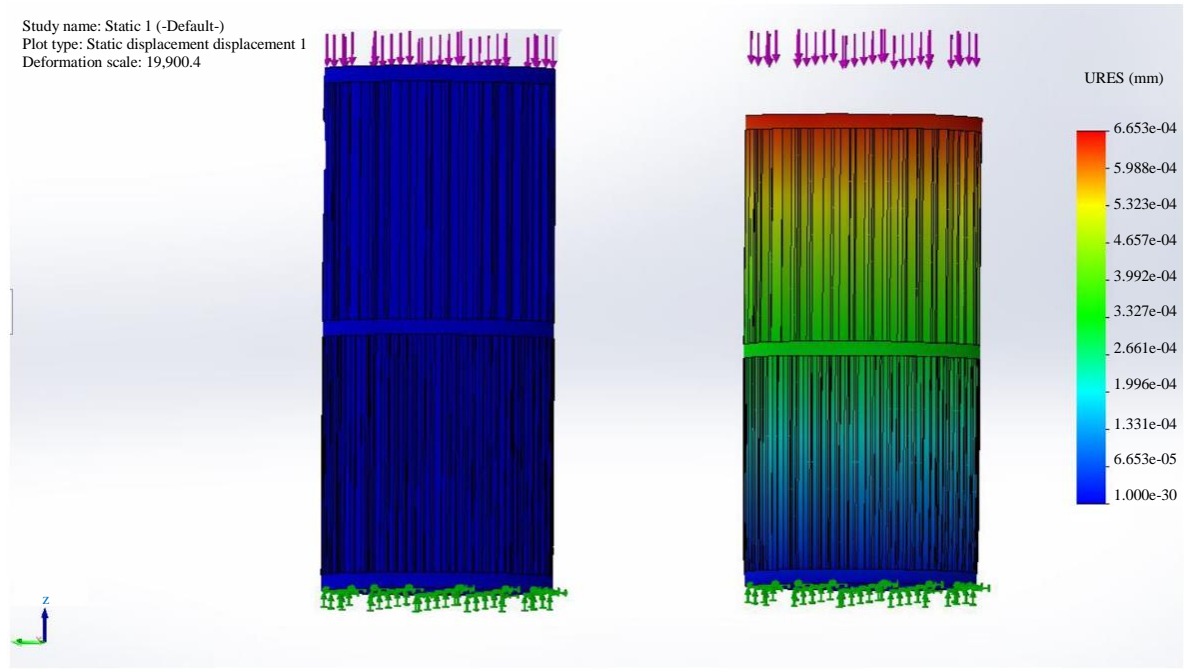

Fig. 15: Displacement Analysis of Honeycomb Cartilage Damper

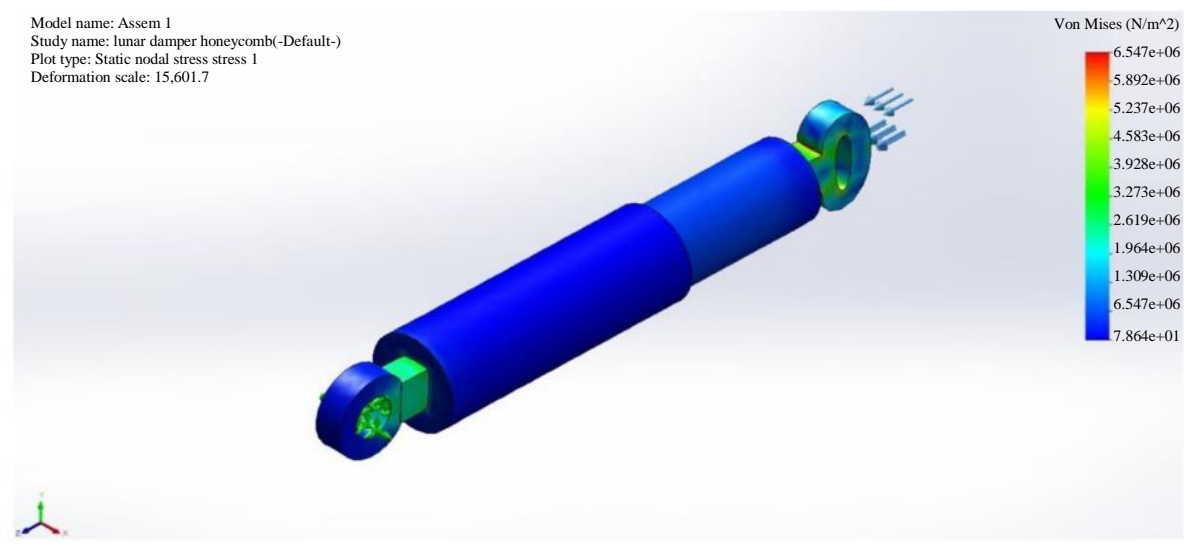

Fig. 16: Static stress analysis of lunar damper

Table 1: Data Observed in FEA

\begin{tabular}{llll}
\hline Name & Type & Min & Max \\
\hline Stresses $(\sigma)$ & Von mises stress & $7.864 \mathrm{e}+01 \mathrm{~N} / \mathrm{m}^{2}$ & $6.547 \mathrm{e}+06 \mathrm{~N} / \mathrm{m}^{2}$ \\
& & Node: 297217 & Node: 297992 \\
Displacement & Resultant displacement & $0.00 \mathrm{~mm}$ & $3.253 \mathrm{e}-03 \mathrm{~mm}$ \\
& & Node: 294178 & Node: 298108 \\
\hline
\end{tabular}

Total static nodal stress of $1500 \mathrm{~N}$ is applied to the damper with fixed geometry (green arrows) and application of force (blue arrows)

\section{Conclusion}

This research work summarizes an investigation of rover perception and the different challenges connected with each since they are applied to the moon examination mission objectives and necessities. Particular challenges are connected with the harsh environments on the moon were presented and discussed along with challenges associated with proposed missions and delivery systems. Hence, a reliable titanium alloy was employed to withstand against said conditions. This study also put forth a notional design strategy to overcome requirements such as reliability. This is a model for comprehension and we can adjust the measurements by planning it on the strength premise. Besides, some essential innovation advancements will be needed to empower tough and exceptionally dependable wanderer framework for longspan missions in brutal planetary conditions.

\section{Author's Contributions}

Anurag Talekar and Ajinkya Dabholkar: coordinated the data-analysis.

Ajinkya Thombe and Karan Jethwa: Contributed to 
the writing of the manuscript.

Nandkumar Bhopale: Designed the research plan and organized the study.

\section{Ethics}

This article is original and contains unpublished material. The corresponding author confirms that all other authors have read and approved the manuscript and no ethical issues have been involved.

\section{References}

Battrick, B., Barron, C., \& Lunar Study Steering Group. (1992). Mission to the Moon. Europe's priorities for the scientific exploration and utilisation of the moon. https://ui.adsabs.harvard.edu/abs/1992mtme.book..... L/abstract

Borowski, S. K., Ryan, S. W., Burke, L. M., McCurdy, D. R., Fittje, J. E., \& Joyner, C. R. (2018). Robust Exploration and Commercial Missions to the Moon Using Nuclear Thermal Rocket Propulsion and Lunar Liquid Oxygen Derived from FeO-Rich Pyroclasitc Deposits.

https://ntrs.nasa.gov/api/citations/20180002979/dow nloads/20180002979.pdf

Carpenter, J. D., Angerer, O., Durante, M., Linnarson, D., \& Pike, W. T. (2010). Life sciences investigations for ESA's first lunar lander. Earth, Moon and Planets, 107(1), 11-23.

https://link.springer.com/article/10.1007\%2Fs1 1038 -010-9375-y

Collier, M. R., Hills, H. K., Stubbs, T. J., Halekas, J. S., Delory, G. T., Espley, J., ... \& Vondrak, R. (2011). Lunar surface electric potential changes associated with traversals through the Earth's foreshock. Planetary and Space Science, 59(14), 1727-1743. https://doi.org/10.1016/j.pss.2010.12.010

Ding, Z., Wu, H., Wang, C., \& Ding, J. (2019). Hierarchical optimization of landing performance for lander with adaptive landing gear. Chinese Journal of Mechanical Engineering, 32(1), 1-12. https://cjme.springeropen.com/articles/10.1186/s100 33-019-0331-0

Glenar, D. A., Stubbs, T. J., McCoy, J. E., \& Vondrak, R. R. (2011). A reanalysis of the Apollo light scattering observations and implications for lunar exospheric dust. Planetary and Space Science, 59(14), 1695-1707. https://doi.org/10.1016/j.pss.2010.12.003
Huang, B., Jiang, Z., Lin, P., \& Ling, D. (2015). Research on impact process of lander footpad against simulant lunar soils. Shock and Vibration, 2015. https://www.hindawi.com/journals/sv/2015/658386/

Huang, M., Nie, H., Zhang, M., Wei, X., \& Yue, S. (2016). Design of mission adaptive landing gear for near space travel lander. Journal of Vibroengineering, 18(8), 4949-4963. https://www.jvejournals.com/article/17078

Jinbao, C., \& Hong, N. (2008). Overloading of landing based on the deformation of the lunar lander. Chinese Journal of Aeronautics, 21(1), 43-47. https://www.sciencedirect.com/science/article/pii/S1 000936108600060

Kosambe, S. (2019). Chandrayaan-2: India's second lunar exploration mission. J. Aircraft and Spacecraft Technology, 3, 221-236. https://doi.org/10.3844/jastsp.2019.221.236

Montgomery, D. C. (1997). The random effects model/Factorial experiments with random factors. Design and analysis of experiments, 4th ed. New York: John Wiley and Sons, 110-117.

Jones, J. (2015) 1971 Apollo Lunar Module LM Operations Handbook, subsystems data and operational procedures. Volumes 1 and 2, US NASA

Nie, T., Gurfil, P., \& Zhang, S. (2019). Bounded lunar relative orbits. Acta Astronautica, 157, 500-516. https://doi.org/10.1016/j.actaastro.2019.01.018

Sahinoz, A. (2012, April). Landing gear design and stability evaluation of a lunar lander for soft landing. In Proceedings of the Bennett conference on mechanical engineering (pp. 1-17).

Wang, C., Nie, H., Chen, J., \& Lee, H. P. (2019). The design and dynamic analysis of a lunar lander with semi-active control. Acta Astronautica, 157, 145-156. https://doi.org/10.1016/j.actaastro.2018.12.037

Yu, M. Y., \& Luo, Y. Z. (2019). Attitude motion path planning on Lyapunov periodic orbits in the circular restricted three-body problem. Acta Astronautica, $163,168-180$.

https://www.sciencedirect.com/science/article/pii/S0 094576519312081 